\title{
INJURY TO THIRD PART OF DUODENUM WITHOUT PANCREATIC INJURY FOLLOWING BLUNT TRAUMA ABDOMEN
}

Mohammed Minhajuddin Harsoori ${ }^{1}$, Shekappa C. Malagimani², K. B. Phulekar ${ }^{3}$

1 Postgraduate, Department of General Surgery, VIMS.

${ }^{2}$ Associate Professor, Department of General Surgery, VIMS.

3Professor, Department of General Surgery, VIMS.

\section{ABSTRACT}

\section{BACKGROUND}

Duodenal perforation following blunt trauma abdomen is extremely rare and can often be overlooked injury due to increased mortality and morbidity. We report a case of injury to second and third part of duodenum without pancreatic injury following blunt trauma abdomen and highlight the changes associated with their management. In this patient, there was a full thickness injury in second and third part of duodenum at laparotomy as he presented with pain abdomen and vomiting. During trauma related laparotomies, complete kocherisation (mobilisation) of the duodenum must be mandatory even in the presence of other injuries. We emphasise on keeping the management protocol simple by doing primary closure of duodenal injury with feeding jejunostomy.

\section{KEYWORDS}

Duodenal Injury, Blunt Trauma Abdomen, Retroperitoneum.

HOW TO CITE THIS ARTICLE: Harsoori MM, Malagimani SC, Phulekar KB. Injury to third part of duodenum without pancreatic injury following blunt trauma abdomen. J. Evolution Med. Dent. Sci. 2016;5(85):6352-6353, DOI: 10.14260/jemds/2016/1435

\section{BACKGROUND}

The duodenum is injured due to crushing or shearing forces on the abdomen. It compromises $0.2-3.7 \%$ of all trauma related laparotomies. The incidence of duodenal injury is $11.2-26 \%$ due to blunt trauma.[1-4] On an average one to four other abdominal injuries are associated with duodenal trauma, which makes an isolated injury a rarity. ${ }^{[4-5]}$

The anatomical relationship of duodenum makes diagnosis and treatment of isolated duodenal injury a difficult task. Due to its rarity and subtle clinical features, the diagnosis and management is often difficult. We described patient with isolated blunt trauma injuries and highlight some points in principles of diagnosis and management of duodenal injury

\section{PRESENTATION OF A CASE}

A 22-year-old male patient met with a road traffic accident and presented with pain abdomen since one day. On clinical examination, patient was found to have tenderness and guarding in all quadrants of abdomen. Bowel sounds were sluggish with distension all over the abdomen. FAST abdomen revealed mild-to-moderate haemoperitoneum. Diagnosis was not conclusive. CT scan of the abdomen was not done, as the patient was not stable. Surgical exploration revealed 1.5 litres of haemoperitoneal fluid and over anti-mesenteric border of duodenum (D2 and D3) a longitudinal laceration of about 7-8 cms (Full thickness injury) was noted. Retroperitoneal bilioma of about 2.4 litres noted. Along with it there was contusion of caecum and ascending colon and bladder wall contusion.

\section{Financial or Other, Competing Interest: None.}

Submission 17-09-2016, Peer Review 11-10-2016,

Acceptance 17-10-2016, Published 24-10-2016.

Corresponding Author:

Dr. Shekappa C. Malagimani,

$\# B / 24$, Staff Quarters,

VIMS (OPD), Cantonment,

Bellary.

E-mail: doc_shekar@yahoo.com

DOI: $10.14260 /$ jemds/2016/1435

\section{(c) (1) $(9)$}

There was no associated pancreatic injury. No perforation of large bowel noted. Primary closure of D2 and D3 full thickness injury was done. Feeding jejunostomy with distal ileostomy was done. Thorough saline wash is given. Duodenum bed drain is secured. Haemostasis is cured. Haemostasis is achieved and dressing done. Patient died on first postoperative day one due to septicaemia.

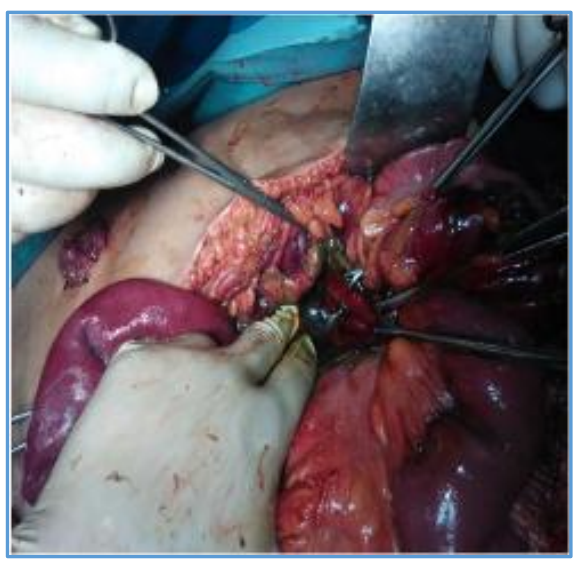

Figure 1. Duodenal Injury

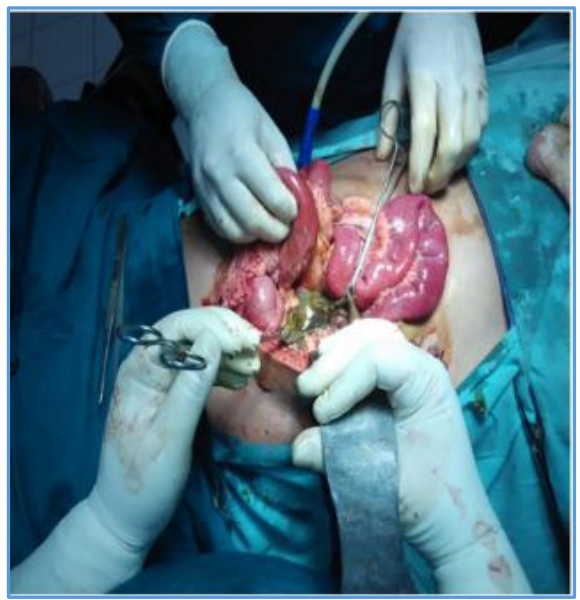

Figure 2. Bile in Lesser Sac 


\section{DISCUSSION}

Because of its retroperitoneal location isolated injuries to the duodenum are uncommon. However, due to this location, patients with injuries to the duodenum after a blunt trauma abdomen are diagnosed late, although more apparent injuries to other organs or vessels are addressed. ${ }^{[1,2]}$ The duodenum is only mobile at the pylorus and its fourth part sharing its blood supply with the pancreas and because of its proximity to the bile duct there is high difficulty in suturing or resecting a segment of the duodenum, especially when the traumatic lesion involves its second part.[3]

Injury to the duodenum following a blunt force can occur either by crushing the duodenum against the rigid vertebral column or due to the impact of shearing forces, as may occur during falls or bursting energy as seen in seat belt injury. ${ }^{[4,5]}$ In our case, the most likely mechanism of injury, based on the information from the site of the accident were the combined effect of crushing and the impact of shearing forces.

Early diagnosis of a patient with a duodenal injury is priceless and the time interval from injury to definitive treatment influences morbidity and mortality. The mortality rate shoots up from $11 \%$ to $40 \%$ if the patients are not explored within $24 \mathrm{hrs}$. ${ }^{[6]}$ Information about the mechanism of injury and physical examination is valuable to suspect duodenal injury. However, the retroperitoneal location of duodenum may preclude early manifestations of injury and physical examinations may be misleading. Retroperitoneal duodenal perforation is usually subtle on presentation, although tachycardia, right upper quadrant tenderness, vomiting and a progressive rise in temperature and heart rate are common findings in patients with these presentations.[7] When our patient was brought to the emergency room he was haemodynamically unstable, presentation with abdominal pain and tenderness on examination and with bilious vomiting. Information about the mechanism of injury combined with clinical findings aroused our suspicion of intraabdominal organ injury, which prompted us for surgical intervention in this patient.

A Computed Tomography (CT) scan of the patient's abdomen with intraluminal and intravenous contrast is the diagnostic test of choice in stable patients with blunt abdominal trauma. ${ }^{[8,9]}$ In our case, the deterioration of the patient's clinical status including bilious vomiting and the inherent high suspicion of abdominal injury indicated the investigation of the intraperitoneal and retroperitoneal space with a CT scan. Although the CT scan could not be performed, ultrasonography findings combined with the clinical findings and the history of the accident increased our suspicion of a possible retroperitoneal duodenal injury. A combined pancreatico-duodenal injury is regarded as a separate category of injury with a high mortality.[10]
In this case, there was no associated pancreatic injury. As the third part of the patient's duodenum was full thickness injury, our choice for reconstruction was limited to primary closure of D2 and D3 full thickness injury with feeding jejunostomy and distal ileostomy done. With this technique, the restoration of duodenal continuity is more physiological and the repair was technically easier. Patient died on 1st postop day due to septicaemia.

\section{CONCLUSION}

Even in this modern era where technology forms a vital part in the diagnosis, clinical judgment of a surgeon based on the history and clinical examination still forms the crux in evaluating a patient with blunt trauma abdomen, especially in cases with duodenal injuries where early intervention makes a significant difference in the outcome of the patient.

\section{REFERENCES}

1. Allen GS, Moore FA, Cox CS, et al. Delayed diagnosis of blunt duodenal injury: an avoidable complication. J Am Coll Surg 1998;187(4):393-9.

2. Fang JF, Chen RJ, Lin BC. Surgical treatment and outcome after delayed diagnosis of blunt duodenal injury. Eur J Surg 1999;165(2):133-9.

3. Asensio JA, Feliciano DV, Britt LD, et al. Management of duodenal injuries. Curr Probl Surg 1993;30(11):1023-93.

4. Boone DC, Peitzman AB. Abdominal injury-duodenum and pancreas. In: Peitzman AB, Rhodes M, Schwab SW, et al. eds. The trauma manual. Philadelphia: Lippincott-Raven 1998. P. 242.

5. Cocke WM, Meyer KK. Retroperitoneal duodenal rupture. Proposed mechanism, review of literature and report of a case. Am J Surg 1964;108:834-9.

6. Lucas CE, Ledgerwood AM. Factors influencing outcome after blunt duodenal injury. J Trauma 1975;15(10):839-46.

7. Carrillo EH, Richardson JD, Miller FB. Evolution in the management of duodenal injuries. J Trauma 1996;40(6):1037-46.

8. Kunin JR, Korobkin M, Ellis JH, et al. Duodenal injuries caused by blunt abdominal trauma: Value of CT in differentiating perforation from hematoma. AJR Am J Roentgenol 1993;160(6):1221-3.

9. Timaran $\mathrm{CH}$, Daley BJ, Enderson BL. Role of duodenography in the diagnosis of blunt duodenal injuries. J Trauma 2001;51(4):648-51.

10. Asensio JA, Buckman RF. Duodenal injuries. In: Ritchie WP. edr. Shackelford's surgery of the alimentary tract. $4^{\text {th }}$ edn. Vol. II. Philadelphia: WB Saunders 1996. P. 110. 